\title{
Recent findings on strong integral input-to-state stability for forced Lur'e systems
}

\author{
by Chris Guiver and Hartmut Logemann
}

We would like to present recent theoretical results on the strong integral Input-to-State Stability (strong iISS) for the following class of finite-dimensional, continuous-time forced Lur'e systems:

$$
\dot{x}=A x+B f(C x)+v, \quad x(0)=x^{0} \in \mathbb{R}^{n} .
$$

Here $A, B$ and $C$ are appropriately sized real matrices, $f$ is a (nonlinear) function, $x$ denotes the state and $v$ is a forcing term (also named, or interpreted, as a disturbance, control or input). Lur'e systems, named after the Soviet scientist A.I. Lur'e, are a common and important class of nonlinear control system, and arise in a number of physically motivated scientific and engineering scenarios, such as the ubiquitous stabilisation of linear systems by saturated static state- or output-feedback.

Strong iISS is a recent stability concept, introduced in [1], and is the conjunction of iISS and ISS with respect to small signals. ISS was introduced in [4] and is the subject of numerous papers including, for example, [6]. ISS is a stability notion with respect to an equilibrium pair, or more general forwardinvariant set, of a (possibly nonlinear) forced control system and, roughly speaking, means that the state has "nice" boundedness properties with respect to initial states and potentially persistent forcing. For nonlinear control systems, ISS has successfully synthesised both state-space and input-output methods resulting in a comprehensive, popular and powerful stability theory.

Integral ISS was introduced in [5] and, again roughly speaking, extends the concept of $L^{p}$ to $L^{\infty}$ stability (for $1 \leq p<\infty$ ) of linear systems to a nonlinear setting, ensuring boundedness of the state when subject to forcing which has "finite energy". ISS implies iISS, but the converse is false in general, so iISS is a weaker concept. The small-signal ISS property is a component of strong ISS which ensures that boundedness of the state has some robustness with respect to small, but potentially persistent, forcing. Quoting [1], strong iISS is an "intermediate property" which has the benefit of "the robustness strengths of ISS and the generality of iISS".

We shall discuss the continued line of enquiry of the authors which asks: to what extent may classical and readily checkable sufficient conditions for absolute stability of the unforced $(v=0)$ Lur'e system $(1)$ be extended to sufficient conditions for stability notions, such as ISS or iISS, of the forced system? Classical absolute stability criteria include the complex Aizerman conjecture [2] and circle criteria [3].

We shall report some preliminary findings of the following nature: if a ball of complex linear static output feedback gains stabilises the underlying linear system (that is, (1) with $f(z)=z$ for all $z$ ), then the zero equilibrium pair of the forced nonlinear Lur'e system (1) is strongly iISS for all $f$ in (1) which satisfy a related "nonlinear" ball condition. The sense in which the nonlinear ball condition holds is crucial for the resulting stability notion which may be inferred. One motivation for the present work is its application to the stabilisation of unstable linear systems by saturated static state- or output-feedback. Such an arrangement naturally leads to a Lur'e system in closed loop. It is known that if $A$ and $f$ in (1) are unstable and bounded, respectively, then under mild assumptions the zero equilibrium pair of (1) is not ISS. We provide sufficient conditions for when such a connection is strongly iISS.

\section{References}

[1] A. Chaillet, D. Angeli, \& H. Ito. Combining iISS and ISS with respect to small inputs: the Strong iISS property. IEEE Trans. Automat. Control, 59 (2014), 2518-2524.

[2] D. Hinrichsen and A. J. Pritchard. Destabilization by output feedback, Differ. Integral Eqn, 5 (1995), $357-386$.

[3] H.K. Khalil. Nonlinear Systems, 3rd ed., Prentice-Hall, Upper Saddle River, 2002.

[4] E.D. Sontag. Smooth stabilization implies coprime factorization, IEEE Trans. Automat. Control, 34 (1989), 435-443.

[5] E.D. Sontag. Comments on integral variants of ISS, Syst. Control Lett., 34 (1998), 93-100.

[6] E.D. Sontag. Input to state stability: basic concepts and results, in P. Nistri and G. Stefani (eds.) Nonlinear and Optimal Control Theory, pp. 163-220, Springer Verlag, Berlin, 2006. 